\title{
Conceptual Design of the Power Supply system for the in-vessel saddle coils for MHD control in ASDEX Upgrade
}

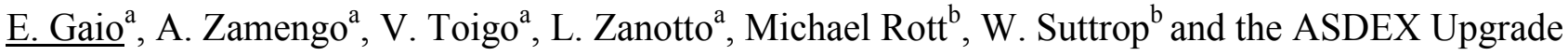 \\ Team \\ ${ }^{\text {a }}$ Consorzio RFX - EURATOM - ENEA Association, C.so Stati Uniti 4, 35127 Padova, Italy \\ ${ }^{\mathrm{b}}$ Max-Planck-Institut für Plasmaphysik, EURATOM Association, D-85740 Garching, Germany
}

In the frame of an ASDEX Upgrade enhancement, a system of active in-vessel coils (of two types, dubbed "A" and "B") and conducting wall for MagnetoHydroDynamic (MHD) stabilization is being designed and implemented.

The conceptual design of the Power Supply (PS) system has been worked out and extensive analyses have been performed to verify that the identified basic modules, based on a cascade topology with two IGBT (Isolated Gate Bipolar Transistor) H-bridges in series for the A units and one H-bridge for the B ones, were able to fulfill all the requirements, well within semiconductors safety operating limits. The paper summarizes the work which led to the definition of the PS requirements, describes the topologies of the selected basic units and of the overall scheme, discusses the design choices and reports the results of the numerical simulations carried out to check the overall performance.

Keywords: ASDEX Upgrade, MHD control, Power Supplies, cascade H-bridges, IGBT

\section{Introduction}

This work was developed in the frame of the Preferential Support Application for an enhancement of the ASDEX-Upgrade (AUG) experiment consisting in the design, procurement and installation of a set of saddle coils and relevant ac power supplies to perform different types of control actions on MHD modes [1]. The enhancement is a joint proposal by four Associations, IPP, FZJ, KTH, and Consorzio RFX, which in particular contributes with its experience in the field of MHD modes control on Reversed Field Pinch [2] and with direct responsibility in the development of the Conceptual Design of the Power Supply System.

MHD instabilities considerably limit the performance of thermonuclear fusion experiments; their control is needed to explore high beta regimes beyond currently achieved parameters. This AUG enhancement represents a strong program on MHD instabilities control, directly addressed to ITER and DEMO needs.

The overall system is designed to perform various control actions and in particular to suppress Edge Localized Modes (ELMs), to control RWMs and to study the effects of establishment of rotating components of the magnetic fields in the control and reduction of the growth rate of Neoclassical Tearing Modes (NTMs) [1].

The active control system is composed of 24 saddle coils, mounted inside the vacuum vessel on the lowfield side and composed of three sets of eight coils distributed toroidally at different poloidal positions (Fig.1), independently fed by power supplies and actively controlled by a real-time system.

The upper and lower set of coils, called " $\mathrm{B}_{\mathrm{U}}$ " and " $\mathrm{B}_{\mathrm{L}}$ " will be provided first, the central coils (called "Acoils"), the conducting wall for RWM stabilization will be added in subsequent stages. The B coil design and electromagnetic features are reported in [3], A-coil design and design verification in [4] and [5], respectively. In this paper the development of the conceptual design of the power supply system is described.

\section{Main requirements for the Active Coils and Power Supplies}

The suppression of ELMs by resonant magnetic perturbation requires the generation of a static (time independent) error field; with the AUG coil system, toroidal mode numbers $n=2, n=3$ or $n=4$ can be produced. The PS are required to produce $1 \mathrm{kA} \mathrm{dc}$ for both A and B coils.

The feedback control for resistive wall mode (RWM) stabilization needs at least $1 \mathrm{mT}$ field produced at the plasma surface at $f=500 \mathrm{~Hz}$ with sufficient phase margin within the useful bandwidth. In terms of PS requirements, the control of RWM instabilities requires an ac current of $440 \mathrm{~A}$ at $500 \mathrm{~Hz}$. Large phase margin implies a fast response of the PS system; should not exceed $100 \mu \mathrm{s}$.

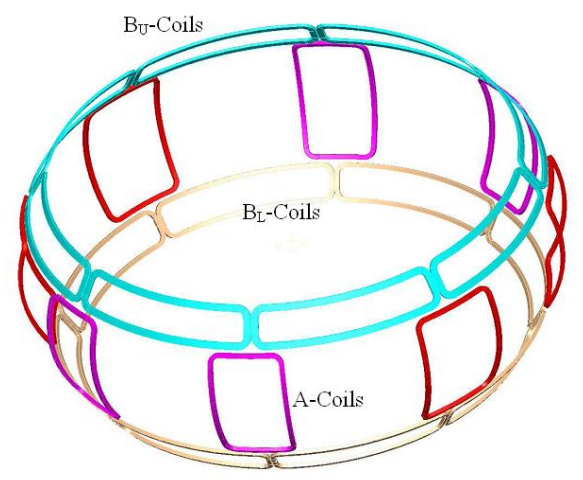

Fig. 1-3D view of the in-vessel coil set

The NTMs control has to be capable to prevent a disruption by locking an existing tearing mode to a rotating error field, this requires that the A coils generate a perturbation field of $3 \mathrm{mT}$, rotating up to $3 \mathrm{kHz}$. The PS 
shall produce an ac current in the coils with amplitude of $1 \mathrm{kA}$ at $3 \mathrm{kHz}$ that corresponds to the most demanding ac specifications.

The PS output voltage values have been defined considering the load impedance (calculated at the maximum operating frequency of the load current) with suitable design margin with respect to that required by the load parameters [3]. Table 1 summarizes the main electrical requirements for the power supplies of $\mathrm{A}$ and $\mathrm{B}$ coils; it can be noted that there are not negligible difference between them.

Table 1 - A-Coils and B-Coils Power Supply electrical requirements

\begin{tabular}{|l|c|c|}
\hline & A-Coils PS & B-Coils PS \\
\hline $\begin{array}{l}\text { Number of } \\
\text { Units }\end{array}$ & 8 & 16 \\
\hline AC operation & $1 \mathrm{kA}^{(1)} @ 3 \mathrm{kHz}$ & $440 \mathrm{~A}^{(1)} @ 500 \mathrm{~Hz}$ \\
\hline DC operation & $1 \mathrm{kA}$ & $1 \mathrm{kA}$ \\
\hline Output Voltage & $\pm 1 \mathrm{kV}$ & $\pm 300 \mathrm{~V}$ \\
\hline Duty Cycle & $10 \mathrm{~s} / 600 \mathrm{~s}$ & $10 \mathrm{~s} / 600 \mathrm{~s}$ \\
\hline
\end{tabular}

(1) peak value of the reference sine wave

\section{Power Supply Design Criteria}

Despite it is still open the evaluation either to select the MHD control in a preprogrammed way or to follow in real time any possible reference corresponding to the control of the instabilities above cited, the last option was assumed for the analyses.

Four quadrant converters implementing fully controllable devices are necessary to cope with the specification of Table 1; IGBT has been evaluated as the most appropriate device. Voltage Source Inverters, composed of ac/dc input stage, dc link capacitors and $\mathrm{dc} / \mathrm{ac}$ bridges were assumed as the basic design approach. The input stage power rating has been optimized by rating the ac/dc converters for the load power dissipation and the capacitor bank in such a way to manage the fast energy exchange with the load.

The selection of the topology, the semiconductor rating and switches modulation law has been derived from an iterative process aimed at reaching a satisfactory compromise among performance, availability, cost and size; the results of this process is summarized in the following. It is pointed out that the development of the conceptual design is aimed at identifying a feasible solution which allows performing the necessary analyses and numerical simulations to verify if the requirements can be fulfilled, to discover possible issues and to be able to better understand and judge the final solution and detailed design that will be proposed by the manufacturer.

The possibility to adopt a full modular solution was considered first because of the advantages in terms of design and test economy, availability, spares, etc, but the overall cost was too high due to the significant differences between the A and B PS requirements (see Table 1). A customized design was therefore followed for A and B PS trying however to push as much as possible the modularity level by identifying a basic module and relevant control which could be suitable for both.

The modulation law and in particular the choice between fix or variable switching frequency was carefully evaluated. Among the large variety of possible considerations on this topic, those related to the semiconductor losses and the noise induced by the switching converters operation were evaluated more deeply. As for the noise, a fixed switching frequency is easier to be filtered while a variable one could be preferable for spreading the noise over a wider range of frequencies leading to lower peak spectral density of the noise power. For the moment, the possibility of a deterministic calculation of the losses led to assume fix switching frequency; this does not exclude possible future revision of the modulation strategies.

\section{Power Supply Design for A and B Coils}

\subsection{A coil PS design}

The first step to address the A coil PS design was the identification of the driver requirement among those summarized in Table 1. Once assumed to adopt fix switching frequency, the most demanding condition is the supply of the nominal dc current which implies the maximum conduction and commutation losses.

A technical solution based on a single $\mathrm{H}$-bridge [6] is not viable because the level of voltage, current and frequency of the output voltage, in the range of tens of $\mathrm{kHz}$ to cope with the bandwidth requirements, imply excessive power dissipation on switching components.

Assuming IGBTs rated for $1.2 \mathrm{kV}$ (size widely used in the market), the voltage requirements for A-coils calls for at least two bridges in series. On this basis the selected scheme to satisfy the A-Coils PS requirements is a cascade with two identical H-bridges in series [7]; the resulting phase voltage applied to the load is the sum of the voltage generated by the two inverters (Fig. 2). This scheme requires independent dc-link capacitors for the $\mathrm{H}$ bridge, each rated for $500 \mathrm{~V}$.

Phase Shifted Carrier PWM (PSCPWM) has been chosen for this PS system [6]. With this type of modulation, each bridge (internally modulated with the 3level modulation technique) shares the same modulation index while the carriers between the bridge inverters are phase shifted by $90^{\circ}$. With this approach, a five level converter has been obtained with a significant improvement of the output frequency, which is four times the switching frequency of the inverter devices.

On the basis of the technical data of available semiconductors, the analyses regarding maximum switching frequency have been carried out assuming natural air cooling and expected system lifetime of 100000 plasma pulses. A switching frequency of $6 \mathrm{kHz}$ can be used with IGBTs rated for $1.8 \mathrm{kA}$. The analyses and numerical simulations of A-coils PS operation showed that the performances required are satisfied with 
safe operation of the devices.

The possibility of adopting Neutral Point Clamp (NPC) topology [7] for A-coil PS was also explored and compared with the cascade one. The details of this study is not reported in this paper, however, among the consideration leading to prefer the cascade solution, there was also the possibility to improve the system modularity because the basic $\mathrm{H}$-bridge of $\mathrm{A}$ unit satisfies the requirements of the B coil PS, as described in the next section.

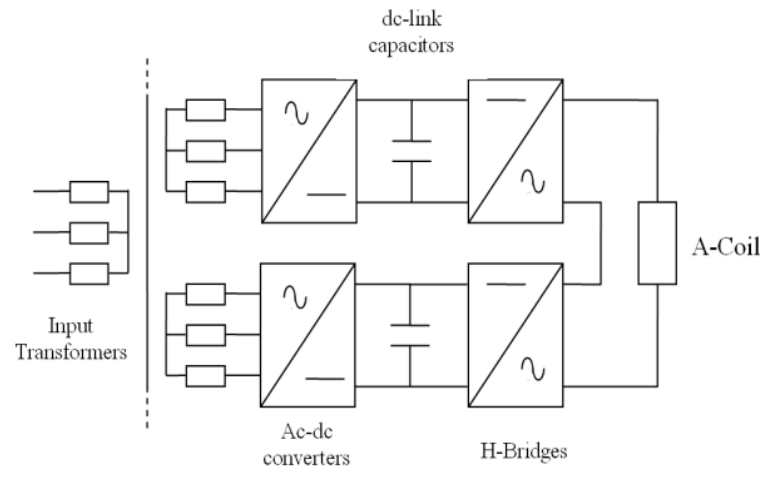

Fig.2 A-Coil Power Supply proposed topology

\subsection{B coil PS design}

One of the two identical modules of the A-Coils PS, with the same dc-link capacitor rated for $500 \mathrm{~V}$, was assumed for the B-coils PS. Its capability to fulfil the requirements of the B-Coils PS reported on Table 1 was verified with numerical simulations. They showed very good performance in terms of ripple, bandwidth and Total Harmonic Distortion (THD) (section 6).

Moreover, for the B-unit this design allows significant margin with respect to the data specified in table 1; this can be useful in particular for the control of the RWM, which in some operational scenarios, can requires a pink-noise shape current on the coils with a bandwidth exceeding $500 \mathrm{~Hz}$.

\section{Overall Power Supply System Scheme}

The overall PS system to feed the 24 new saddle coils of ASDEX results a quite modular system composed of 32 modules as shown in Fig.3: 16 power modules will be used to feed the 16 B-Coils ( 8 " $\mathrm{B}_{\mathrm{U}}$ " and 8 " $\mathrm{B}_{\mathrm{L}}$ ") and 16 power modules to feed the 8 A-Coils (two modules in series for each coil).

The total input power is $1.6 \mathrm{MW}(50 \mathrm{~kW}$ times 32 separated ac sources); this ratings permit to sustain the load power dissipation and, together with about $10 \mathrm{mF}$ $\mathrm{dc}$ link capacitors, to fulfil the system requirements in all the foreseen operating scenarios.

Multisecondary cast resin transformer has been selected as the reference technology for the PS input section. Fig. 3 shows the cheapest solution with a single transformer composed of one primary and 32 secondary windings. To improve the system availability, but with impact on cost and size, two or three independent transformers (one for A and the others for B coils) could be provided. The transformer primary side is fed by the local $10 \mathrm{kV}$ grids supplied by one out of three AUG flywheel generator or, alternatively, by the public $10 \mathrm{kV}$ power grid.

An important aspect of the PS design is whether the dc-link voltage needs to be regulated in real time or not. When the PSs are required to supply a dc current, the possibility of reducing the dc link voltage allows reducing the ripple of the output current and this has an important benefit. Nevertheless in this condition the control system request of fast reaction to a growing plasma instability could not be satisfied because the input $\mathrm{ac} / \mathrm{dc}$ rectifier takes at least some ms to re-charge the dc link and the available output voltage could be not enough to assure the required current variation. Thus it was chosen to always maintain the dc link at the nominal value, leaving to the inverter the regulation of output voltage. This assumption allows simplifying the input scheme: a diode rectifier instead of thyristor rectifiers can be chosen; this input scheme can be selected also because the expected range of variation of the ac input voltage is not too high and because the rectifier power rating is quite limited, thus simplifying the start-up circuit which is typically composed of a pre-charger resistor shortcircuited by contactors.

In conclusion, the complete base topology of each power module is composed of transformer secondary, a diode bridge rectifier, a capacitor bank with a pre-charger circuit and an inverter H-bridge

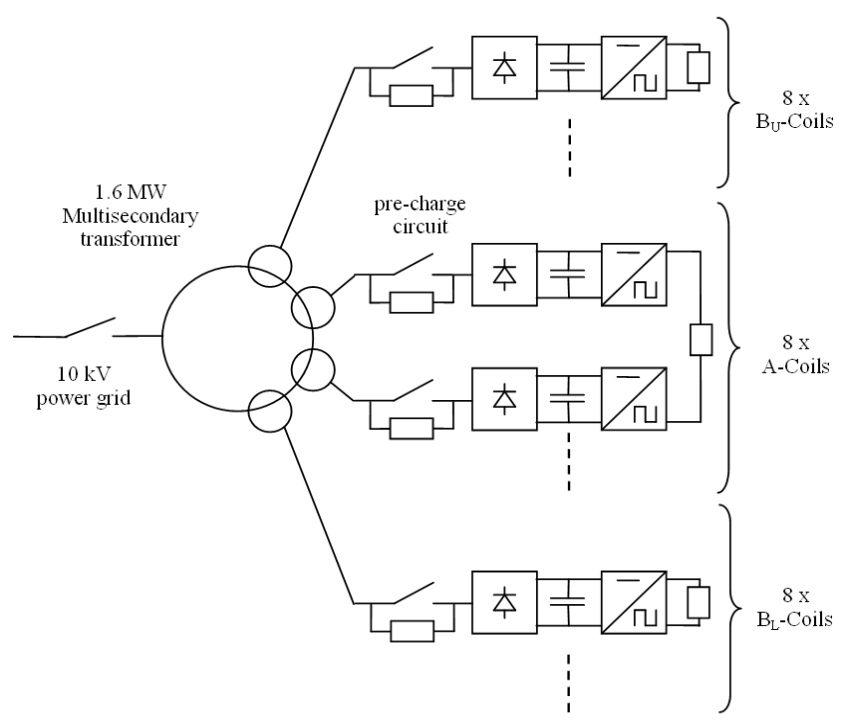

Fig. 3 Overall scheme of A and B coils Power Supply

The evaluation of the PS grounding point has been carried out considering the machine constraints (limited space nearby the coils feedthroughs doesn't permit the grounding at machine side), the PS topology and the effects on the induced noises on the nearest electronic devices. The analyses are still ongoing and are also related to the design of an optional EMI filter which can be used to reduce the amplitude of the common mode stray currents. 


\section{Models Simulations}

The operation of the Power Supplies of the A-Coils and B-Coils proposed in this paper were simulated in order to verify the capability to satisfy the requirements. Analyses have been performed with PSIM ${ }^{\circledR}$; the current references have been selected to be similar to the expected waveforms during the experiments: $\mathrm{dc}$ operation to mitigate ELM activity, ac operation to generate rotating field to control NTM and RWM and pink-noise waveform to simulate the RWM scenario.

In Figure 4 is shown the $3 \mathrm{kHz}$ ac scenario of the ACoils PS, the 5-levels voltage output and the good reproduction of the sine wave can be recognized (the THD, is below 2\%). Figure 5 shows the similar operation of B-coils PS with the sinusoidal reference waveform $500 \mathrm{~Hz}-440 \mathrm{~A}$; the three-level output voltage is sufficient in this case to produce the sine wave. Table 2 summarizes details of the simulation results: current ripple has been evaluated with $1 \mathrm{kA} \mathrm{dc}$ on the load, rise time is the time needed to reach $1 \mathrm{kA}$ in case of current step waveform reference, THD has been calculated in ac scenario at the maximum operating frequency.

Table 2 -Simulation results

\begin{tabular}{|c|l|c|}
\hline & & $\begin{array}{c}\text { Simulation } \\
\text { results }\end{array}$ \\
\hline \multirow{4}{*}{$\begin{array}{c}\text { A-Coils } \\
\text { PS }\end{array}$} & Current Ripple $@ 1 \mathrm{kA}$ & $\pm 0,75 \%$ \\
\cline { 2 - 3 } & Rise Time & $\approx 70 \mu \mathrm{s}$ \\
\cline { 2 - 3 } & THD $@ 3 \mathrm{kHz}, 1 \mathrm{kA}$ & $1,56 \%$ \\
\hline \multirow{3}{*}{$\begin{array}{c}\mathrm{B}_{\mathrm{L}} \text {-Coils } \\
\text { PS }\end{array}$} & Current Ripple & $\pm 1,7 \%$ \\
\cline { 2 - 3 } & Rise Time & $\approx 120 \mu \mathrm{s}$ \\
\cline { 2 - 3 } & THD @ $500 \mathrm{~Hz}, 440 \mathrm{~A}$ & $0,78 \%$ \\
\hline \multirow{3}{*}{$\begin{array}{c}\mathrm{B}_{\mathrm{U}} \text {-Coils } \\
\text { PS }\end{array}$} & Current Ripple $@ 1 \mathrm{kA}$ & $\pm 1,3 \%$ \\
\cline { 2 - 3 } & Rise Time & $\approx 120 \mu \mathrm{s}$ \\
\cline { 2 - 3 } & THD $@ 500 \mathrm{~Hz}, 440 \mathrm{~A}$ & $1,10 \%$ \\
\hline
\end{tabular}

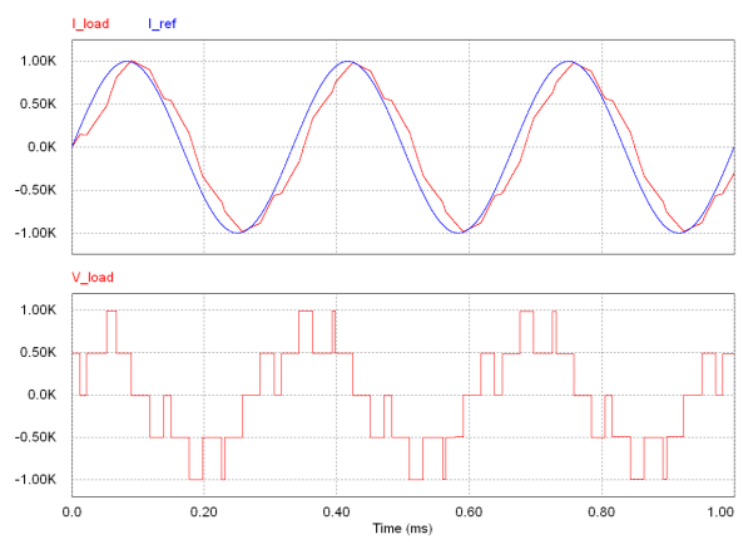

Fig. 4 - A-Coils PS operation with $3 \mathrm{kHz}-1 \mathrm{kA}$ sinusoidal reference waveform. On the first plot the blue line represents the current reference while the red one the simulated current on the load. On the second plot the simulated load voltage.

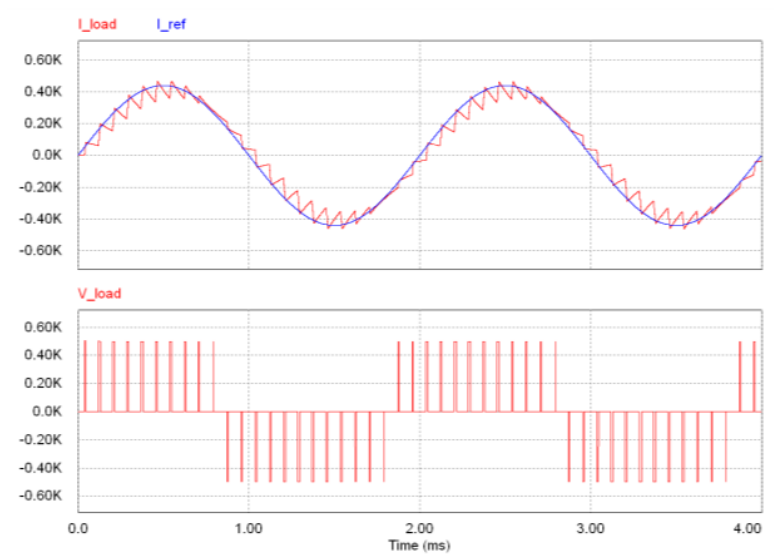

Fig. $5-\mathrm{B}_{\mathrm{U}}$-Coils PS operation with $500 \mathrm{~Hz}-440 \mathrm{~A}$ sinusoidal reference waveform. On the first plot the blue line represents the current reference while the red one the simulated current on the load. On the second plot the simulated load voltage.

\section{Conclusions and future works}

The conceptual design of the PS system for the invessel saddle coils for MHD control in ASDEX Upgrade has been presented. The proposed scheme is customized for the different requirements relevant to the specified controls for A and B coils but, at the same time, it has a sufficient modularity degree, adopts components widely used in the market and presents a very good ratio between performance and cost/size limitation. Further work is in progress to analyse the effect of different location of the grounding points and schemes for EMI filters to mitigate the common mode stray currents.

\section{References}

[1] W. Suttrop, et. alt, "In-vessel saddle coils for MHD control in ASDEX Upgrade", Fusion Engineering and Design, Volume 84, Issues 2-6, June 2009, pp 290-294.

[2] P. Sonato, et al., Machine modification for active MHD control in RFX, Fusion Engineering and Design 66-68 (2003), 161-168

[3] M. Rott, U. Seidel, B. Streibl, W. Suttrop, T. Vierle, "Electro-magnetic modeling of the planned active in-vessel coils at ASDEX Upgrade", Fusion Engineering and Design, Volume 84, Issues 7-11, June 2009, pp 1653-1657

[4] T. Vierle et al, "PEEK-insulated conductors of fast invessel saddle coils for ASDEX Upgrade" this conference

[5] I. Zammuto et al, "Conceptual design of in vessel midplane saddle coils for fast ac operation in ASDEX Upgrade" this conference

[6] V. Toigo, E. Gaio, N. Balbo, A. Tescari, The power supply system for the active control of MHD modes in RFX, Fusion Engineering and Design, Vol. 66-68, Sep. 2003, pp 1143-1147

[7] J.Rodriguez, Jih-Sheng Lai, Fang Z. Peng "Multilevel inverters: a survey of topologies, controls, and applications" IEEE Transactions on Industrial Electronics,, Vol. 49, 2002, pp. 724-738. 